\title{
Frailty as a Nexus Between the Biology of Aging, Environmental Conditions and Clinical Geriatrics
}

\author{
Luigi Ferrucci, $\mathrm{MD}, \mathrm{PhD},{ }^{1}$ \\ Charles Hesdorffer, MBBCh, MMED, ${ }^{1}$ \\ Stefania Bandinelli, MD, ${ }^{2}$ \\ Eleanor M. Simonsick, $\mathrm{PhD}^{1}$
}

\begin{abstract}
Chronic diseases often determine pathologic phenotypes similar to those traditionally attributed to aging, such as accelerated decline of muscle mass and increments of basic metabolic rate, suggesting that the true nature of aging is progressively increasing entropy in the face of failing homeostatic mechanisms. Aging in different animal species and in humans suggest that increasing entropy causes major problems in four domains; body composition, energetic imbalance between availability and demand, homeostatic dysregulation, and neurodegeneration. In humans, loss of integrity and function in these domains causes manifestations similar to frailty, especially if the damage is severe and/or involves multiple domains, and has catastrophic consequences, such as physical and cognitive disability. Characterizing these phenotypes, and understanding the mechanisms by which they emerge with increasing entropy is a necessary step to find interventions that can prevent, delay or moderate the effects of aging. Pharmacological and non-pharmacological interventions that may effectively modulate the aging phenotypes are actively studied and will certainly be ready in the near future. Until then, creating a "senior friendly society", that allows maximal independence but also promotes an active and healthy lifestyle may be the most cost-effective intervention to improve the quality of life in the population.
\end{abstract}

Key words: Frailty, aging, older adults

Recommended Citation: Ferrucci L, Hesdorffer C, Bandinelli S, Simonsick EM. Frailty as a Nexus Between the Biology of Aging, Environmental Conditions and Clinical Geriatrics. Public Health Reviews. 2010;32:475-88.

\footnotetext{
${ }^{1}$ Longitudinal Studies Section, Clinical Research Branch, National Institute on Aging, NIH, Baltimore, MD, USA

${ }^{2}$ Geriatric Department, ASF - Firenze, Florence, Italy
}

Corresponding Author Contact Information: Luigi Ferrucci at ferruccilu@grc.nia.nih.gov; Longitudinal Studies Section, Clinical Research Branch, National Institute on Aging, NIA, ASTRA Unit, Harbor Hospital, 5th Floor, 3001 S. Hanover Street, Baltimore, MD 21225-1233 


\section{INTRODUCTION}

At the societal level seemingly spontaneous adaptations to aging occur more rapidly than scientists and policy makers can respond to them. These changes involve fundamental aspects of life including how individuals look towards their future, education, legislation, health, social security and family dynamics. New ideas that permeate the "structural" aspects of social organization in the areas of architectural design, city planning, transportation, employment and the working environment, are highly influenced by the demographic transformation. In particular, our city landscape is evolving to include communities built around the special needs of older persons, with an unprecedented wide range of choices. Interestingly, while these profound changes in society implicitly recognize that there is something different about the 'older person', which is so fundamental that it requires a significant readjustment in society, the basic information needed to understand and direct these changes is presently missing. How can we in a regimen of limited resources make society more "age friendly" without alienating younger generations and avoid the intergenerational conflicts that are looming on the horizon?

This article argues that to plan a "senior friendly society" necessitates that we acquire a more in depth understanding of the multidimensional factors that affect the aging process and of the mechanisms by which the environment, social relationships, and disease interact with aging to slow down or accelerate the development of frailty. Unless modern society can prevent some of the burden of disability associated with age-related frailty, the rise in health and social security costs will not be sustainable ${ }^{1}$ because it will progressively wear down their competiveness in the world market and challenge the stability of their economies. ${ }^{2}$

\section{AGING, DISEASE AND THE FAILURE OF HOMEOSTATIC MECHANISMS}

The study of aging as a medical specialty is a relatively recent phenomenon. In spite of its youth, ideas and techniques developed by the "aging" researcher have already been adopted by other specialties because they seem to describe unexpected consequences of chronic diseases in a rapidly aging population. ${ }^{3}$ For the past one hundred years, the focal point of research in medicine has been classification of disease, with an emphasis on defining the differential diagnosis of a specific disease process. More recently, however, evidence has emerged suggesting that many chronic 
diseases cause changes in the human body that are similar to those traditionally attributed to aging. Diabetes, chronic pulmonary diseases, HIV, cancer and renal failure are associated with accelerated decline of muscle mass, relative increase in adipose tissue and increments of basic metabolic rate. These changes probably reflect the extra-energy required to maintain homeostatic equilibrium in the face of entropy due to disease. ${ }^{4,5}$ Interestingly, a decline in lean body mass and a dysregulated metabolic rate are also traditionally described as two of the main phenotypes of aging and are the focus of intensive studies in the aging field. Thus, it appears that the end-stage of many disease processes and aging share the same phenotypes and perhaps similar mechanisms.

The relationship between basic aging phenotypes and the manifestation of some of the most pervasive chronic diseases is puzzling. For decades scientists claimed that aging and disease were distinct processes and, therefore, should be approached independently. Although this view was essential to characterize "normal" and recognize that aging and disease are not synonymous, this perspective lacks empirical support. In fact, the more we learn about aging the more it becomes clear that aging and disease have similar manifestations and similar underlying mechanisms.

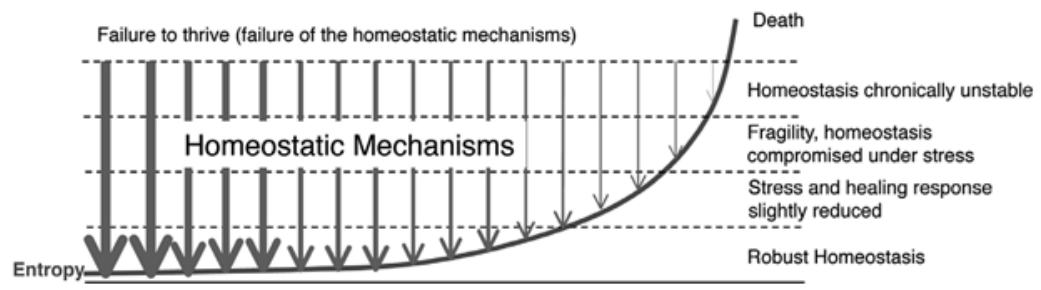

Fig. 1. Aging may be conceptualized as a process of progressively increased entropy coupled with reduction and subsequently failure of the homeostatic mechanisms.

The graph in Figure 1 illustrates how the true nature of aging may reflect the underlying trajectory of increased entropy, which also relates to disease development. Note that here for simplicity frailty is treated as a linear process, while the true functional form of frailty is probably stepwise and non-linear. At the time of conception, favorable environmental conditions and hormonal signaling facilitate the well-organized and structured growth of an organism. Entropic pressure exists, but is counterbalanced by very robust and energetically expensive homeostatic mechanisms. During childhood, the consequences of increasing entropy 
are not evident because they are continuously counteracted by similar mechanisms. However, if one or only a few of the homeostatic mechanisms fail, entropic forces prevail and a phenotype of accelerated aging, even in a child, may become apparent. Werner's syndrome, the quintessential progeroid syndrome of premature aging, is emblematic of this process. ${ }^{6}$ Progeroid syndromes represent a window through which we can peer at the consequences of relatively unrestrained entropy on human physiology. This viewpoint presupposes, of course that aging constitutes an entropic phenomenon. ${ }^{7}$

In the absence of disease, in youth and middle age, there is homeostatic redundancy, which provides remarkable resistance to stress and capacity to heal and recover. Infectious diseases may be managed by the immune system without even becoming fully symptomatic. Bone fractures heal in a few days. Staying up until the wee hours can be followed by a relatively productive day at work. Chronic diseases interrupt this well-oiled machine - homeostatic resources become challenged. As a result, additional energy above that typically required for homeostasis is needed to oppose the progression of entropy. Just like chronic diseases, the aging process can slowly and progressively diminish homeostatic resources and reduce the capacity to cope with internal and environmental stress. For an older person, an upper respiratory infection can quickly turn into pneumonia. A bone fracture only partially calcifies after weeks or months. A night in the hospital can precipitate disorientation or even severe delirium. These are just a few examples of how small environmental stressors can cause very severe problems in older persons. In the final stages of life, homeostatic resources may be inadequate to maintain a state of equilibrium even at rest, in the absence of stressors, leading to the well-described syndrome of "failure to thrive".

If both aging and disease affect health by depleting and damaging homeostatic resources, it should come as no surprise that they both tend to cause similar, stereotypic phenotypes. Such phenotypes are manifestations of the natural entropic evolution of the human "machine" no longer protected by homeostatic forces. Understanding aging phenotypes is key to identifying social and clinical environment that bolster and support essential function in the face of failing homeostatic mechanisms. Ways of preventing and managing age-related frailty through medical interventions and creating environmental supportive circumstances that can add new functional abilities to the nearly exhausted homeostatic mechanisms. But first, we need to accomplish three goals: 
1) Develop a theoretical framework and testable hypotheses of the overarching nature of the basic aging phenotypes, with the assumption that they are caused by increasing entropy;

2) Design models that describe the progression of impairments in basic homeostatic mechanisms that allow for a large degree of initial redundancy, followed by progressive and acute catastrophic threats to this redundancy. The expected outcome would be initial maintenance of a functional existence, replaced over time by irreversible frailty and rapid progression to disability and death;

3) Develop testable hypotheses on how medical, behavioral and environmental interventions may support the homeostatic forces that counteract frailty, disability and death.

\section{FRAILTY AND THE AGING PHENOTYPES}

Frailty has been defined as a "physiological syndrome characterized by decreased reserve and diminished resistance to stressors, resulting from cumulative decline across multiple physiological systems, causing vulnerability to adverse outcomes and high risk of death." ${ }^{3}$ While having a definition is appealing and this one in particular underscores the multidimensional nature of the syndrome, converting this concept into an operational definition used by scientists and clinicians has in practice proved difficult and raised considerable controversy. ${ }^{8}$

Among the different definitions in the literature, the syndromic characterization, proposed by Fried and colleagues, which combines information on weight loss, fatigue, impaired grip strength, diminished physical activity and slow gait has both excellent internal consistency and a strong predictive validity. ${ }^{9-12}$ Ken Rockwood and Mitnitski have suggested that frailty cannot be defined within the boundaries of a syndrome because of the nature of the random accumulations of multiple impairments that occur with aging. ${ }^{13}$ It is difficult to refute the intuitive attractiveness of a definition based on "random accumulation of deficits" because of its strong face validity. However, such a view implies little hope of the possibility of prevention and treatment. In addition, the appeal for the "random" theory of frailty is somewhat lost in translation. Ken Rockwood, who first proposed this theory, suggested that a frailty index could be constructed using information commonly collected in geriatric practice. ${ }^{14}$ We believe that this translational approach limits the detection of frailty to a very late stage, when interventions are unlikely to be effective. 
In the genesis of most definitions of frailty proposed in the literature, one senses a conflict between the need to use data already collected in large clinical and epidemiological studies versus the need to describe the mechanisms that cause frailty in older individuals. Indeed, current definitions largely use information on overt manifestation of frailty, such as a reduction in strength, lower extremity performance and mobility. Such definitions discriminate only the most severe cases, when all possible compensation and compensatory strategies are already curtailed, and despite activation of these mechanisms, substantial deterioration in physical and cognitive function has become evident. If we want to dissect the process that leads to frailty, we need to shift focus toward changes that occur at an earlier stage when the basic causal mechanisms are still detectable and potentially reversible.

Aging in different animal species and in humans, show universal and consistent patterns in four major domains; body composition, energetic imbalance between availability and demand, homeostatic dysregulation, and neurodegeneration. In humans, loss of integrity and function in these domains causes manifestations similar to frailty, especially if the damage is severe and/or involves multiple domains. It is reasonable to hypothesize that the consequences of loss of integrity and function are invariant, whether the cause of it is aging itself, disease, environmental pressure or stress.

A hypothetical model of the relationship between aging, the aging phenotypes, frailty and geriatric syndromes is illustrated in Figure 2. Profound changes in body composition may be the most evident and inescapable effects of aging. Body weight increases from birth until late middle age and then declines between age 65 and 70 in men, and somewhat later, in women. Lean body mass decreases steadily after the third decade, while fat mass tends to increase in middle age and then declines in late life. Sex hormone levels decrease with age in both men and women. Most aging individuals, even those who remain healthy and fully functional, tend to develop a mild proinflammatory state characterized by high levels of proinflammatory markers, including interleukin-6 (IL-6) and C-reactive protein (CRP), and signs of increased damage from oxidative stress. Deficiency of some micronutrients, such as vitamins (especially vitamin D), minerals (selenium and magnesium) and antioxidants (carotenoids and vitamin E), also affect homeostatic capacity. Fitness, estimated as peak oxygen consumption (MVO2 peak) during an exercise test, declines with aging and the consequences of such decline are more severe in individuals who are sedentary and in those affected by chronic diseases. Resting Metabolic Rate, the amount of energy expended at rest, declines with 
aging, due to a decline in lean body mass and tends to increase (or decline less) in individuals with multiple chronic conditions. The combination of these two trends with age requires sick older people to consume most of their available energy to perform essential activities of daily living. Neurons stop reproducing shortly after birth and their numbers decline throughout life. Studies have suggested that brain and spinal cord atrophy may contribute to declines in cognitive and motor function often observed with aging. Clinically, cortical and sub-cortical changes are reflected in the high prevalence of "soft", nonspecific neurological signs, which manifest as a slow and unstable gait, poor balance and slow reaction times. $^{15}$

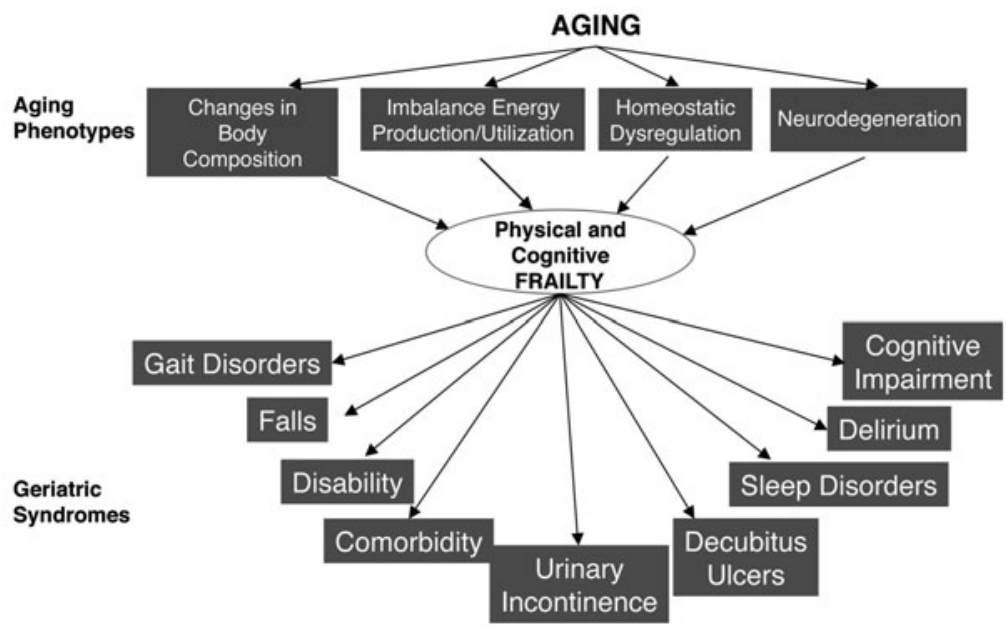

Fig. 2. Descriptive model of the relationship between aging, the aging phenotypes and the geriatric syndromes.

Conceptualizing frailty through the four main underlying processes changes in body composition, energetic imbalance, homeostatic dysregulation, and neurodegeneration - recognizes that the processes that underlie frailty start at birth, but progress more rapidly later in life, with a high degree of heterogeneity between individuals. ${ }^{16}$ Perhaps, even more important, this approach provides common criteria by which aging, disease and environmental pressure, contribute to the "aging phenotype" and, in turn, to frailty. For example, if you examine chronic diseases within the context of how they impact the four aging phenotypes, you will better 
understand the interface between chronic disease and frailty: heart failure causes low energy availability, is associated with multiple hormonal derangements, and a proinflammatory state, and thus contributes to the severity of frailty. Parkinson's disease affects body composition, energy metabolism and homeostatic signaling; resulting in a syndrome that closely resembles frailty. Ultimately, it becomes apparent that almost all chronic diseases highly prevalent in old age fit this model.

It is useful to explore how the aging phenotypes participate in the determination of frailty and the so-called "geriatric syndromes", which are generally considered the cornerstones of geriatric medicine. A number of studies suggest that frailty is a powerful risk factor for most geriatric syndromes, such as sleep disorders, anorexia and malnutrition, chronic pain, incontinence, falls, skin breakdown, gait disorders, depression and loss of mobility. ${ }^{17,18}$ However, the causal link between frailty and geriatric syndromes remains descriptive and observational rather than mechanistic. Interestingly, most "geriatric syndromes" can be viewed as deriving from specific combinations of over-expressed aging phenotypes. For example, delirium is often precipitated by an environmental stress, typically hospitalization. Its nature, as an expression of neurodegenerative-induced fragility is suggested by the fact that cognitive impairment and dementia are strong risk factors for the development of delirium during a stressful condition or circumstance. ${ }^{19}$ Urinary incontinence in older individuals is usually due to a combination of reduced muscle mass/strength, and altered neurological reflexes, possibly related to neurodegeneration and unresolved chronic infections. These examples illustrate how the aging phenotypes represent the nexus, interconnecting aging, chronic diseases, frailty and "geriatric syndromes". They also represent a possible model for clinical reasoning and diagnosis in geriatric practice. For example, the emergence of urinary incontinence should trigger a diagnostic work up that, beyond the symptom of incontinence itself, addresses the larger issues of critically diminished skeletal muscle mass and strength.

But, are aging phenotypes and their relationship with chronic diseases modifiable? Although the evidence for this possibility is thin, it is certainly conceivable that at some point in the near future, pharmacologic and nonpharmacologic treatments will be available that could effectively counteract the body composition changes, energetic, hormonal dysregulation, proinflammatory state, excessive oxidative stress and neurodegeneration in individuals with accelerated aging and/or substantial comorbidity, hopefully by enhancing and supporting the natural homeostatic mechanisms that oppose the progression to frailty. Whether intervening at these levels can 
truly slow down the progression of entropy with aging is presently unknown, but clearly needs to be tested. Little is known about the homeostatic mechanisms that counteract entropy. By observing how compensation occurs with aging, we should be able to hypothesize the structure of the building blocks of these homeostatic mechanisms and their mode of function.

\section{HOMEOSTATIC MECHANISMS: STRUCTURE AND PROGRES- SIVE IMPAIRMENT}

Given the critical role of homeostatic mechanisms, evolutionary selection likely facilitated the creation of substantial redundancy and the selection of such redundancy was aimed at maximizing the chance of reproduction. However, such redundancy also allowed substantial life span after the reproductive state.

The conceptualization of redundant homeostatic mechanisms is illustrated in Figure 3. Let's postulate that maintenance of homeostasis requires at least some integrity of the different homeostatic mechanisms. For simplicity, the example in Figure 3 only shows four systems.

A simple array of homeostatic mechanisms that serves different domains has scarce redundancy and possibilities to adapt and, therefore, does not fit the highly adaptive model of frailty development that we have previously described (top of Figure 3A). In this simple model, partial function of the homeostatic mechanism/s is unlikely to be effective and, therefore, once one single mechanism becomes dysfunctional, the overall stability of the entire mechanism is lost. The system is intrinsically fragile and unlikely to survive to reproduction.

The bottom of Figure 3A, demonstrates an alternative, redundant, adaptive system. There are multiple complementary homeostatic mechanisms for each single domain. Only a few (one in the example) are active at rest, but others may be recruited when environmental or other stressors exceed a given threshold (stress response), or when the main homeostatic mechanism becomes ineffective or completely dysfunctional. The redundant system maintains function even in the face of cumulative damage, as long as such damage is not concentrated on a single mechanism but, rather, is distributed at random, as expected by increasing entropy (Figure 3B). This hypothetical system buffers the progressive dysfunction of regulatory mechanisms and is less likely to reach the critical point when there are not enough resources to ensure stability in the absence of stress, but fail to maintain homeostasis in the presence of over stressors. Perhaps 
this example best describes frailty. It then becomes apparent how finally, even such a redundant system will fail when the accumulation of damage is so massive and diffuse (as described in Figure 3C), or is concentrated in one domain as may occur in a specific disease (Figure 3D).
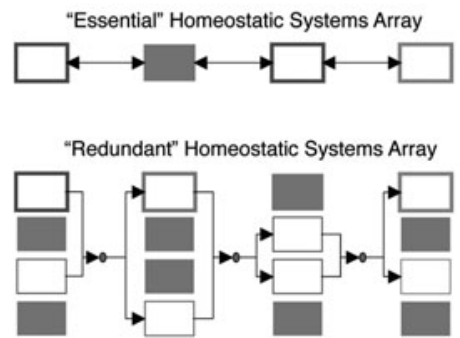

A

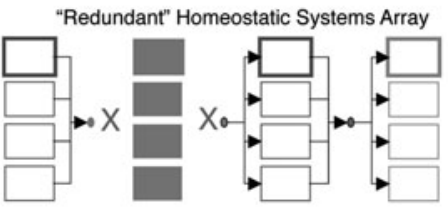

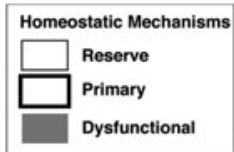

"Redundant" Homeostatic Systems Array

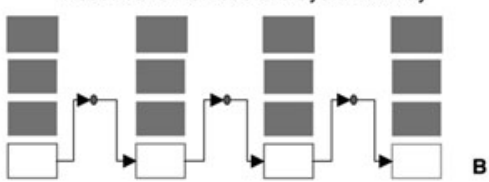

"Redundant" Homeostatic Systems Array

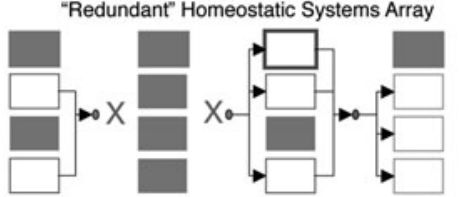

D

Fig. 3. Hypothetical models of homeostatic redundancy systems. See text for explanation.

One critical question concerning this model, is whether periodic stressing of the homeostatic mechanisms makes them more robust or weak. The principle of 'hormesis' suggests that a continuous subliminal stimulation may be positive and reinforces stress resistance..$^{20}$ On the other hand, there are instances of homeostatic mechanisms becoming exhausted by excessive demand leading to their failure. For example, chronic anemia is initially compensated by the over production of Erythropoietin (EPO), the compensatory effect fails briskly when this over-production of EPO can no longer be sustained..$^{21}$ Analogously, in the initial phase of type 2 diabetes, the overproduction of insulin maintains glycemic control, but is only temporarily effective because it rapidly exhausts the capacity of beta-cells to produce insulin. Assessing the strength of compensatory resources is crucial to any strategy aimed at preventing frailty. The study of these homeostatic mechanisms thus requires longitudinal investigation studies that are specifically directed, long-term and high-tech in order to devise the various combinations and permutations that may be involved in the intricate 
process that results in frailty as the outcome of aging. Substantially more difficult will be to develop studies that include the concepts of how the environment and society influence the evolution of the aging phenotypes and thus affect the risk of developing frailty.

\section{TOWARD AN “AGING FRIENDLY” SOCIETY}

We are accustomed to think about homeostatic mechanisms in biological terms. However, specific behavioral and environmental factors may empower physiological homeostatic mechanisms and, perhaps, also supplement new homeostatic mechanisms when the original ones become dysfunctional. In Figure 3 we hypothesized that multiple homeostatic mechanisms function in parallel to allow redundancy and improved response to stress. Some of these mechanisms can be thought to be behavioral or environmental in nature.

In some instances, the role of environmental factors in reducing frailty is immediately evident. At the beginning of the last century, the availability of clean water, abundant food, shelter, heating and cooling systems, relief from hard labor, and (perhaps) antibiotics created the possibility for increasing longevity and prevented frailty more than all the drugs, medical devices, diagnostic techniques or any other interventions invented to date. ${ }^{22}$ In fact, recent studies suggest that progress in medical science has mostly reduced mortality, with no or little effect on active life expectancy and the quality of life in old age. ${ }^{23}$ Understanding whether and how less basic aspects of the environment/behavior can affect the development of frailty and the quality of aging is less obvious. This is mostly an uncharted area filled with conjecture and hypotheses.

Can we imagine a society that implements policy aimed at counteracting the aging phenotypes, preventing frailty and improving the quality of life in old age? Gerontologists are hopeful that making a society "aging friendly" might be the most powerful tool we have to cope with the challenges imposed by the demographic swing to longevity. Note that the term "aging friendly" is used here to signify not only a social and living environment that removes barriers to an independent and successful life for older persons, but also a society that creates legislation, sets environmental goals and plans and establishes incentives aimed at promoting behavior that counteracts the emergence of the aging phenotypes. Thinking about ways to slow down the development of the aging phenotype and moderate their impact on global function, we could imagine a society that encourages physical activity, not only in the form of recreational exercise or hobbies, 
but as an integral part of everyday living in communities; a society, that by leveraging all available human resources, stimulates a purposeful life even in older and disabled individuals; a society that focuses on productivity, but also values relaxation and personal growth; a society that stimulates healthy nutrition by providing the incentives for the production and consumption of fruit, vegetables and other healthy food free from contaminants; a society that supports interaction within and between families and communities.

Compiling an exhaustive list of the interventions that may positively affect the aging phenotypes would make an interesting exercise. The list would inevitably be much shorter if all the ideas needed to be cost-effective. Even scientifically testing the effectiveness of these interventions in modifying the trajectories of the aging phenotypes, prolonging active life expectancy, and delaying physical and cognitive disability would be prohibitively expensive. However, not pursuing such a course of action is not a viable option. Medical care can reduce mortality, but seems to do very little to improve physical and cognitive function in old age - the factors that burden individuals and predict healthcare resource utilization and cost. Until we find the secret to slowing down the biological clock of aging and preventing the development of dementia and other disabilities characteristic of frailty we should start to promote "healthy aging", based on our presently, rather incomplete, knowledge of the "aging process". Reshaping our society in response to the demographic transformation may be the most powerful strategy to accomplish this goal.

Aknowledgments: supported in part by the Intramural Research Program of the National Institute on Aging, NIH, Baltimore, MD. The author would like to thank Megan Homer for her precious editorial suggestions.

Conflicts of Interest: None declared.

\section{REFERENCES}

1. Louria DB. Extraordinary longevity: Individual and societal issues. J Am Geriatr Soc. 2005;53:S317-9.

2. Anderson GF, Hussey PS. Population aging: A comparison among industrialized countries. Health Aff (Millwood). 2000;19:191-203.

3. Walston J, Hadley EC, Ferrucci L, Guralnik JM, Newman AB, Studenski SA, et al. Research agenda for frailty in older adults: Toward a better understanding of physiology and etiology: Summary from the American Geriatrics Society/ National Institute on Aging Research Conference on Frailty in Older Adults. J Am Geriatr Soc. 2006;54:991-1001.

4. Glass D, Roubenoff R. Recent advances in the biology and therapy of muscle wasting. Ann N Y Acad Sci. 2010;1211:25-36. 
5. Ruggiero C, Metter EJ, Melenovsky V, Cherubini A, Najjar SS, Ble A, et al. High basal metabolic rate is a risk factor for mortality: The Baltimore Longitudinal Study of Aging. J Gerontol A Biol Sci Med Sci. 2008;63:698706.

6. Kipling D, Davis T, Ostler EL, Faragher RG. What can progeroid syndromes tell us about human aging? Science. 2004;305:1426-31.

7. Ershler WB, Ferrucci L, Longo DL. Hutchinson-Gilford progeria syndrome. N Engl J Med. 2008;358:2409-10.

8. Bergman H, Ferrucci L, Guralnik J, Hogan DB, Hummel S, Karunananthan S, Wolfson C. Frailty: An emerging research and clinical paradigm--issues and controversies. J Gerontol A Biol Sci Med Sci. 2007;62:731-7.

9. Fried LP, Tangen CM, Walston J, Newman AB, Hirsch C, Gottdiener J, et al. Frailty in older adults: Evidence for a phenotype. J Gerontol A Biol Sci Med Sci. 2001;56:M146-56.

10. Fried LP, Xue QL, Cappola AR, Ferrucci L, Chaves P, Varadhan R, et al. Nonlinear multisystem physiological dysregulation associated with frailty in older women: Implications for etiology and treatment. J Gerontol A Biol Sci Med Sci. 2009;64:1049-57.

11. Fried LP, Hadley EC, Walston JD, Newman AB, Guralnik JM, Studenski S, et al. From bedside to bench: Research agenda for frailty. Sci Aging Knowledge Environ. 2005;2005(31):pe24.

12. Bandeen-Roche K, Xue QL, Ferrucci L, Walston J, Guralnik JM, Chaves P, et al. Phenotype of frailty: Characterization in the women's health and aging studies. J Gerontol A Biol Sci Med Sci. 2006;61:262-6.

13. Rockwood K, Mitnitski A. Frailty defined by deficit accumulation and geriatric medicine defined by frailty. Clin Geriatr Med. 2011;27:17-26.

14. Rockwood K, Hubbard R. Frailty and the geriatrician. Age Ageing. 2004;33:42930.

15. Ferrucci L, Bandinelli S, Cavazzini C, Lauretani F, Corsi A, Bartali B, et al. Neurological examination findings to predict limitations in mobility and falls in older persons without a history of neurological disease. Am J Med. 2004;116:807-15.

16. JW Rowe and RL Kahn. Human aging: Usual and successful. Science 1987;237:143-149.

17. Inouye SK, Studenski S, Tinetti ME, Kuchel GA. Geriatric syndromes: Clinical, research, and policy implications of a core geriatric concept. J Am Geriatr Soc. 2007;55:780-91.

18. Tinetti ME, Inouye SK, Gill TM, Doucette JT. Shared risk factors for falls, incontinence, and functional dependence. Unifying the approach to geriatric syndromes. JAMA. 1995;273:1348-53.

19. Young J, Inouye SK. Delirium in older people. BMJ. 2007;334:842-6.

20. Gems D, Partridge L. Stress-response hormesis and aging: "That which does not kill us makes us stronger”. Cell Metab. 2008;7:200-3. 
21. Ferrucci L, Guralnik JM, Woodman RC, Bandinelli S, Lauretani F, Corsi AM, et al. Proinflammatory state and circulating erythropoietin in persons with and without anemia. Am J Med. 2005;118:1288.

22. Bongaarts J. Human population growth and the demographic transition. Philos Trans R Soc Lond B Biol Sci. 2009;364:2985-90.

23. Crimmins EM, Beltrán-Sánchez H. Mortality and morbidity trends: Is there compression of morbidity? J Gerontol B Psychol Sci Soc Sci. 2011;66:75-86. 